\section{Gerinnungsfaktor IV}

\section{T. Stief}

Institut für Laboratoriumsmedizin und Pathobiochemie, Krankenhaus der Philipps-Universität, Marburg, Deutschland

Synonym(e) Calcium-Ionen; $\mathrm{Ca}^{2+}$

\section{Englischer Begriff $\mathrm{Ca}^{2+}$}

Definition > Calcium ist ein chemisches Element mit dem Elementsymbol $\mathrm{Ca}$ und der Ordnungszahl 20; 99 \% des im Körper vorkommenden Calciums befinden sich in Zähnen und in Knochen, aus letzteren kann Calcium bei Calciummangel freigesetzt werden und steht dann für andere Aufgaben zur Verfügung. So kommt Calcium wesentliche Funktionen bei der Erregung von Muskeln und Nerven, beim
Glykogenstoffwechsel, im Komplementsystem, und nicht zuletzt bei der Blutgerinnung zu.

Beschreibung Erst wenn N-terminale Glutaminsäurereste Vitamin-K-abhängiger - Gerinnungsfaktoren (FII, FVII, FIX, FX = F2, F7, F9, F10) posttranslational mittels einer weiteren Carboxylgruppe zu $\gamma$-Carboxyglutaminsäureresten (Gla) modifiziert werden, können sie $\mathrm{Ca}^{2+}$ binden. Die Bindung von $\mathrm{Ca}^{2+}$ ändert die Konformation der Gerinnungsfaktoren, welches die Bindung an Phospholipid-(PL-)Membranen z. B. von Thrombozyten erleichtert. Calcium ist wesentlicher Bestandteil der drei wichtigsten membrangebundenen Enzymkomplexe der Gerinnung (intrinsische Tenase: F9a-F8a-PL-Ca ${ }^{2+}$; extrinsische Tenase: F7a-TF [tissue factor]-PL-Ca ${ }^{2+} ;$ F10a-[Prothrombinase-]Komplex: F10a-F5a-PL-Ca ${ }^{2+}$ ). 